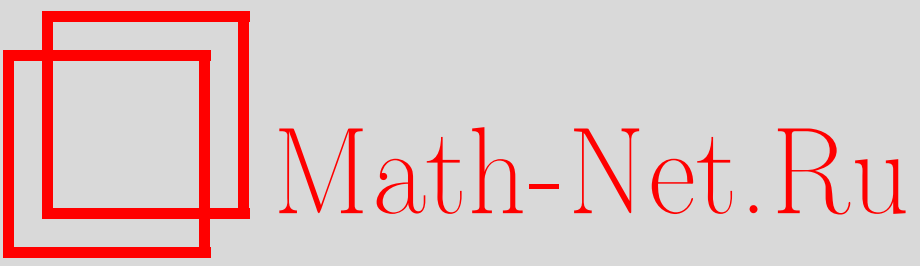

А. Н. Ширяев, Минимаксная оптимальность метода кумулятивных сумм (CUSUM) в случае непрерывного времени, УМH, 1996, том 51, выпуск 4, 173-174

DOI: https://doi.org/10.4213/rm1010

Использование Общероссийского математического портала Math-Net.Ru подразумевает, что вы прочитали и согласны с пользовательским соглашением

http://www.mathnet.ru/rus/agreement

Параметры загрузки:

IP : 52.23 .180 .231

26 апреля 2023 г., 12:49:23 


\title{
МИНИМАКСНАЯ ОПТИМАЛЬНОСТЬ МЕТОДА КУМУЛЯТИВНЫХ СУММ (CUSUM) В СЛУЧАЕ НЕПРЕРЫВНОГО ВРЕМЕНИ
}

\author{
А. Н. ШиРяЕв
}

1. Предполагается, что на некотором вероятностном пространстве $(\Omega, \mathscr{F}, \mathrm{P})$ наблюдается случайный процесс $X=\left(X_{t}\right)_{t \geqslant 0}$ со стохастическим дифференциалом

$$
d X_{t}=r I(t-\theta) d t+\sigma d w_{t}, \quad X_{0}=0,
$$

где $w=\left(w_{t}\right)_{t \geqslant 0}-$ стандартный винеровский процесс, $I(x)=\left\{\begin{array}{ll}1, & x \geqslant 0, \\ 0, & x<0 .\end{array}\right.$ Момент $\theta \in[0, \infty]$ интерпретируется как момент появления "разладки" (по терминологии [1]-[4]) в ходе наблюдений за процессом $X$ : при $t<\theta$ наблюдаемый процесс $X_{t}=\sigma W_{t}$, а при $t \geqslant \theta$ процесс $X_{t}=X_{\theta}+r(t-\theta)+\sigma\left(W_{t}-W_{\theta}\right)$.

Будем через $\tau=\tau(\omega)$ обозначать момент подачи сигнала о появлении "разладки", т.е. пусть $\tau$ есть марковский момент со значениями в $[0, \infty]$ относительно потока $(\mathscr{F} t)_{t \geqslant 0}$, где $\mathscr{F}_{t}=\sigma\left\{\omega: X_{s}, s \leqslant t\right\}, t \geqslant 0$.

В наших работах [1]-[3] рассматривались различные постановки задачи обнаружения и были найдены оптимальный байесовский метод и оптимальный метод в предположении, что разладка появляется "на фоне установившегося стационарного режима наблюдения".

В настоящей работе доказывается минимаксная оптимальность непрерывной версии метода кумулятивных сумм (CUSUM), введенного Пэйджем [5], для следующего критерия, предложенного Лорденом [6].

Пусть $\mathrm{P}_{t}$ - распределение вероятностей процесса $X$ в предположении, что момент разладки $\theta=t$. Распределение $\mathrm{P}_{\infty}$ отвечает случаю отсутствия разладки $(\theta=\infty), \mathrm{P}_{0}$ соответствует случаю наличия разладки в момент $\theta=0$. Обозначим $\mathfrak{M}_{T}=\left\{\tau: \mathrm{E}_{\infty} \tau=T\right\}$-класс моментов остановки $\tau$, для которых среднее время $\mathrm{E}_{\infty} \tau$ до подачи (ложной) тревоги равно $T$.

Качество системы наблюдения, идентифицируемой с выбором момента $\tau \in \mathfrak{M}_{T}$, будем оценивать величиной $R(T ; \tau)=\sup _{\theta \geqslant 0} \operatorname{ess} \sup _{\omega} \mathrm{E}_{\theta}\left([\tau-\theta]^{+} \mid \mathscr{F}_{\theta}\right)$. Момент $\tau_{T}^{*} \in \mathfrak{M}_{T}$ естественно называть оптимальным (в минимаксном смысле), если $R\left(T ; \tau_{T}^{*}\right)=\inf _{\tau \in \mathfrak{M}_{T}} R(T ; \tau)$.

Теорема. При каждом $T>0$ в классе $\mathfrak{M}_{T}$ существует оптимальный момент $\tau_{T}^{*}$ mакой, что $\tau_{T}^{*}=\inf \left\{t \geqslant 0: \gamma_{t} \geqslant B_{T}^{*}\right\}$, где $\gamma=\left(\gamma_{t}\right)_{t \geqslant 0}, \gamma_{t}=\max _{\theta \leqslant t} \frac{d \mathrm{P}_{\theta}}{d \mathrm{P}_{\infty}}(X, t)$, есть “CUSUM"-прочесс; $\frac{d \mathrm{P}_{\theta}}{d \mathrm{P}_{\infty}}(X, t)$ - производная Радона-Никодима сужения $\mathrm{P}_{\theta} \mid \mathscr{F}_{t}$ мерь $\mathrm{P}_{\theta}$ относительно сужения $\mathrm{P}_{\infty} \mid \mathscr{F}$ меры $\mathrm{P}_{\infty}$ на $\mathscr{F}$.

При әтом $\gamma_{t}=\max _{\theta \leqslant t} \frac{L_{t}}{L_{\theta}}$, где $L_{t}=\exp \left(\frac{r}{\sigma^{2}} X_{t}-\frac{r^{2}}{2 \sigma^{2}} t\right) ;$ порог $B_{T}^{*}$ определяетсл (в предположсении $\frac{r^{2}}{2 \sigma^{2}}=1$, что не ограничивает общности) как корень уравнения $T=B-1-\ln B ;$ минимаксный риск $R\left(T ; \tau_{T}^{*}\right)=\mathrm{E}_{0} \tau_{T}^{*}=\ln B_{T}^{*}-1+\frac{1}{B_{T}^{*}} ;$ при больиих $T$ $R\left(T ; \tau_{T}^{*}\right)=\ln T-1+O\left(\frac{1}{T}\right) ;$ прим мальх $T \quad R\left(T ; \tau_{T}^{*}\right)=T+O\left(T^{2}\right)$.

ЗАмечАнИЕ. В случае дискретного времени вопрос об оптимальности метода CUSUM рассматривался в работах Мустакидеса [7] и Ритова [9]. Асимптотическая оптимальность этого метода была доказана в работе Лордена [6]. См. также сборник статей [8], который посвящен проблематике наискорейшего обнаружения "разладки" (иначе - "change-point") в разнообразных постановках. Рассматриваемый нами случай непрерывного времени является (в сравнении с работами [7], [9], относящимися к случаю дискретного времени) несколько более деликатным в техническом отношении (по причине необходимости оперирования с феноменами типа локалного времени), но зато позволяет получить также и точные форомулы, которые дают достаточно хорошие аппроксимации в моделях с дискретным временем.

Как и в [7] одним из ключевых моментов в доказательстве является получение оценки снизу для величин $R(T ; \tau)$ (ср. (3) с (8) в [7]). 
2. Доказательство теоремы опирается на следующие результаты.

Лемма 1. Процесс $\gamma=\left(\gamma_{t}\right)$ является решением неоднородного стохастического дифференциального уравнения $d \gamma_{t}=\frac{r}{\sigma^{2}} \gamma_{t} d X_{t}+d H_{t}, \gamma_{0}=1$, где $H_{t}=-\int_{0}^{t} I\left(\gamma_{s}=1\right) \frac{d M_{s}}{M_{s}} c$ $M_{s}=\min _{\theta \leqslant s} L_{\theta}$.

Решение уравнения (12) может быть представлено в виде

$$
\gamma_{t}=L_{t}\left\{1+\int_{0}^{t} \frac{d H_{s}}{L_{s}}\right\}
$$

Представление (2) играет существенную роль при доказательстве следующего утверждения.

ЛЕмма 2. Для любого конечного момента остановки $\tau$

$$
\sup _{\theta \geqslant 0} \underset{\omega}{\operatorname{essup}} \mathrm{E}_{\theta}\left([\tau-\theta]^{+} \mid \mathscr{F}_{\theta}\right) \geqslant \frac{\mathrm{E}_{\infty} \int_{0}^{\tau} \gamma_{t} d t}{\mathrm{E}_{\infty} \gamma_{\tau}} .
$$

Лемма 3. В классе $\mathfrak{M}_{T}$ момент $\tau_{T}^{*}$ обладает одновременно следующими двумя свойствами оптимальности:

$$
\inf _{\tau \in \mathfrak{M}_{T}} \mathrm{E}_{\infty} \int_{0}^{\tau} \gamma_{t} d t=\mathrm{E}_{\infty} \int_{0}^{\tau_{T}^{*}} \gamma_{t} d t, \quad \sup _{\tau \in \mathfrak{M}_{T}} \mathrm{E}_{\infty} \gamma_{\tau}=\mathrm{E}_{\infty} \gamma_{\tau_{T}^{*}}
$$

ЛЕмма 4. Для момента $\tau_{T}^{*} R\left(T ; \tau_{T}^{*}\right)=\mathrm{E}_{0} \tau_{T}^{*} u$

$$
\mathrm{E}_{\infty} \int_{0}^{\tau_{T}^{*}} \gamma_{t} d t=B_{T}^{*} \log B_{T}^{*}+1-B_{T}^{*}, \quad \mathrm{E}_{0} \tau_{T}^{*}=\ln B_{T}^{*}-1+\frac{1}{B_{T}^{*}}
$$

ДОКАЗАТЕЛЬСТВО ТЕОРЕМЫ. ИЗ Лемм 2 и 4

$$
\mathrm{E}_{0} \tau_{T}^{*}=R\left(T ; \tau_{T}^{*}\right) \geqslant \inf _{\tau \in \mathfrak{M}_{T}} R(T ; \tau) \geqslant \frac{\inf _{\tau \in \mathfrak{M}_{T}} \mathrm{E}_{\infty} \int_{0}^{\tau} \gamma_{t} d t}{\sup _{\tau \in \mathfrak{M}_{T}} \mathrm{E}_{\infty} \gamma_{\tau}}
$$

В силу леммы 4 левая часть и правая часть в (4) оказьваются равными, что и доказывает утверждение теоремы.

\section{СПИСОК ЛИТЕРАТУРЫ}

[1] Ширяев А. Н. // ДАН СССР. 1961. Т. 138. №4. С. 794-801. [2] Ширяев А. Н. // ДАН СССР. 1961. Т. 138. № 5. С. 1039-1042. [3] Ширяев А. Н. // Теория вероятн. и ее примен. 1963. T. VIII. № 1. С. 26-51. [4] Ширяев А. Н. Статистический последовательный анализ. M.: Наука, 1976. [5] Page E. S. // Biometrika. 1954. V. 41. P. 100-115. [6] Lorden G. // Ann. Math. Statist. 1971. V. 42. P. 1897-1908. [7] Moustakides G. V. // Ann. Statist. 1986. V. 14. P. 1379-1387. [8] Carlstein E., Müller H.-G., Siegmund D. (ed. ) Change-point problems // Institute of Math. Statist. Lecture Notes - Monograph Ser. V. 23, 1994. [9] Ritov Y. // Ann. Statist. 1990. V. 18. P. 1464-1469. 